\title{
Alain A. Grenier (dir.), Tourisme polaire
}

dossier de la revue Téoros, avril 2009, 100 pages

\section{Philippe Violier}

\section{(2) OpenEdition}

1 Journals

Édition électronique

URL : http://journals.openedition.org/tourisme/295

DOI : $10.4000 /$ tourisme.295

ISSN : 2492-7503

Éditeur

Éditions touristiques européennes

\section{Édition imprimée}

Date de publication : 1 décembre 2010

Pagination : 112-114

ISSN : 2109-5671

\section{Référence électronique}

Philippe Violier, « Alain A. Grenier (dir.), Tourisme polaire », Mondes du Tourisme [En ligne], 2 | 2010, mis en ligne le 30 septembre 2015, consulté le 22 septembre 2020. URL : http://journals.openedition.org/ tourisme/295; DOI : https://doi.org/10.4000/tourisme.295

Ce document a été généré automatiquement le 22 septembre 2020.

\section{cc) (†)}

Mondes du tourisme est mis à disposition selon les termes de la licence Creative Commons Attribution - Pas d'Utilisation Commerciale - Pas de Modification 4.0 International. 


\section{Lectures critiques}

\section{Alain A. Grenier (dir.), Tourisme polaire}

dossier de la revue Téoros, avril 2009, 100 pages

\section{Philippe Violier}

\section{RÉFÉRENCE}

Alain A. Grenier (dir.), Tourisme polaire, revue Téoros, vol. 28, n 1, Presses de l'université du Québec, avril 2009.

1 La revue Téoros livre, dans son numéro 1 de l'année 2009, un dossier thématique consacré au "tourisme polaire". Le dossier a été dirigé par Alain Grenier, professeur à l'université du Québec à Montréal, qui inaugure en même temps ses nouvelles fonctions de directeur et rédacteur en chef de la revue.

2 La première contribution, "Conceptualisation du tourisme polaire, cartographier une expérience aux confins de l'imaginaire" est due à Alain GRENIER. La diversification des pratiques par laquelle l'auteur a choisi d'aborder la question montre toute la richesse des expériences vécues et complique singulièrement la tâche qu'il s'est fixé.

3 L'entreprise de délimitation de l'objet, bienvenue et documentée, et qui s'appuie manifestement sur une connaissance intime, n'est cependant pas complètement satisfaisante. Tout d'abord, le géographe sera surpris de l'image que le sociologue renvoie de la géographie. En effet, après une première approche historique et un inventaire des "produits" ou des "activités", voire des "formes", l'auteur esquisse une conceptualisation binaire, qui passe par la mobilisation de ce qu'il nomme la géographie et s'achève par une approche dite sociologique. Alain Grenier borne alors la géographie à un exercice de délimitation spatiale et de descriptions dignes des pionniers de la discipline, découvreurs et autres explorateurs. Il ignore visiblement les travaux conduits par des géographes sur le rôle des représentations dans le tourisme, 
depuis Cazes, Debarbieux ou encore Knafou... ou ceux qui ont tenté d'établir une relation entre les individus et les lieux (Équipe Mit). Au-delà, s'il n'est pas douteux que le touriste joue à l'explorateur lorsqu'il choisit de se rendre dans ces contrées, nous voyons mal comment la dimension spatiale, la destination, pourrait être absente du concept. Du coup, le sens échappe un peu : n'est-ce pas un bout du monde... peuplé ? L'auteur cite d'ailleurs des touristes évoquant le vide... La mobilisation de l'analyse systémique pouvait permettre d'associer toutes les dimensions dont l'auteur donne un aperçu fouillé à travers des tableaux dont la construction n'est pas toujours explicitée (tableau 3). Il manque également un plaidoyer en faveur du choix du qualificatif "polaire" par rapport à la ribambelle des tourismes de quelque chose, utilisés par d'autres auteurs et que l'auteur cite. Certes, la tâche a été accomplie semble-t-il par Grenier lui-même, mais dans une revue plutôt spécialisée (Polar Geography). Le mot polaire renvoie assez directement à l'"expédition polaire", qui est l'un des ressorts, fort bien souligné par l'auteur, de cette découverte des extrêmes ; mais, du coup, ce mot ne colle pas avec la totalité de la panoplie des pratiques. Par ailleurs, qu'est-ce qui justifie de rassembler dans une même catégorie l'Antarctique, continent dépourvu d'habitants permanents, et les terres septentrionales de l'hémisphère Nord ? En quoi le tourisme polaire est-il autre chose qu'une subdivision de la subdivision qui prend place dans le tableau positiviste des sciences, au bout du bout? Y aurait-il un tourisme tropical, un tourisme des déserts, des ergs, des regs ou de la rose des sables?

L'article de Denis JALLAT, "Le tourisme polaire et sa construction dans l'histoire" restreint un peu le champ. L'auteur y analyse, en effet, comment s'est construit, dans les années 1920 et 1930, un imaginaire polaire lié à la conquête des dernières terres inconnues, qui a préparé la mise en tourisme. Il s'agit là de la variante polaire d'un processus plus général et plus ancien à l'origine des pratiques touristiques les plus fréquentes, comme le repos au bord de la mer ou les promenades en montagne. La science comme l'art ont été de puissants facteurs de la diffusion du tourisme dans le monde ; avec les pôles, la sphère est bouclée. S'ajoutent, là comme ailleurs, les enjeux géopolitiques qui consistent à manifester, par des mobilités, une présence dans des terres lointaines. Ces rappels n'auraient pas été inutiles pour replacer cette dynamique dans un contexte plus vaste et pour montrer que ce qui se joue là n'est en rien original.

L'ensemble est intéressant et nous informe. L'évocation des projets de détournement des courants marins polaires pour refroidir les tropiques est savoureuse, mais le corpus de textes utilisé n'est pas assez présenté : il est question d'une revue Je sais tout au sujet de laquelle l'auteur n'écrit presque rien, et qui n'est pas cité en bibliographie, mais aussi de bon nombre d'autres ouvrages, romans ou de journaux comme L'Illustration, citée au contraire... Combien d'articles consacrés aux pôles dans cette période ? Était-ce un sujet "à la une" ? Le propos en est un peu affaibli.

Dans "The polar cultural heritage as a tourism attraction. A case study of the airship mooring mast at Ny-Ålesund, Svalbard", Ricardo ROURA étudie le rôle du marker, en l'occurrence l'histoire de la conquête des pôles, matérialisée par le mât d'amarrage des dirigeables de l'expédition Amundsen et Nobile (1926), dans la construction de l'attraction touristique. Il revisite ainsi les approches de MacCannell et Neil Leiper sur le système touriste-sight-marqueur. L'auteur écrit lui-même que, sans le discours des guides, les touristes passeraient sans s'en préoccuper devant ce mât de ferraille qui servait à ficeler les dirigeables, voire en pestant contre les criminels qui n'hésitent pas à abandonner, même aux pôles dont la blancheur manifeste la pureté la 
plus éclatante, des amas de ferraille témoins, d'un usage dépassé. Une photo prise par l'auteur montre que d'ailleurs, lorsque passent des bélugas, les touristes n'écoutent plus rien et photographient les animaux. À qui rendre hommage? Aux touristes bien intentionnés, qui n'hésitent pas à parcourir ces terres extrêmes à la recherche de témoignages parcimonieux ? ou aux guides qui continuent malgré tout à y croire?

7 Sophie DUPRÉ signe avec "Les croisières touristiques dans l'Arctique canadien. Une réalité tangible à l'appropriation territoriale encore limitée" un article très bien documenté, solide et passionnant. L'auteur, après avoir explicité clairement son objet, a ensuite exploité une base de données liée à la surveillance et à la sécurité de la navigation dans la zone (Nordreg établie depuis 1974). Cela lui permet de retracer l'évolution des croisières et à en montrer la croissance, qui est très progressive et mesurée. Elle évalue la fréquentation de l'Arctique canadien à 3000 personnes par an et, du coup, relativise fortement l'appropriation des lieux par les touristes. Elle analyse également les types de lieux mis en tourisme en distinguant les sites étapes et les villages à fonction touristique. Au final, quatre lieux sont visités. Tout cela est bien moins que la présence militaire au temps de la guerre froide ou que la population des bases scientifiques.

8 Nous avons été moins convaincu par l'évocation des risques, abordée à la fin de l'article, parce que cette approche reste globale - des risques pour qui ? Mais, puisque il s'agit de l'objet de la recherche de thèse de Sophie Dupré, cet article subtil nous incline à penser que cela n'est que partie remise.

9 L'article de Véronique ANTOMARCHI, “Tourisme, identité et développement en milieu inuit. Le cas de Puvirnituq au Nunavik", éclaire un autre angle du tourisme dans ces régions. Le tourisme est abordé depuis un lieu fréquenté, Puvirnituq, étudié sous les angles de la découverte de la culture, de la patrimonialisation et du rôle des habitants dans la mise en tourisme. L'auteur s'appuie sur un travail de terrain fouillé et produit une réflexion argumentée et intelligente. Les villageois attendent du tourisme une valorisation de leur culture et des retombées économiques afin de fournir des emplois aux jeunes, puisque la croissance démographique est forte. Si l'analyse des leviers est bien menée, et notamment de la formation de la population ou l'implication des autorités autonomes, pour que le tourisme permette d'atteindre les objectifs de développement durable, pour une fois appréhendé dans sa globalité, y compris son versant économique, nous ne suivons pas l'auteur qui semble ranger dans les bonnes pratiques l'exercice du monopole aérien et qui recommande, afin d'éviter des “jalousies", une régulation par répartition des touristes qui n'a pas fait ses preuves ailleurs. En effet, une seule compagnie aérienne permet d'accéder à ce village distant de 1900 kilomètres de Montréal. Elle est contrôlée par les autochtones, ce qui semble vertueux, sauf que les tarifs sont prohibitifs et limitent singulièrement le nombre d'arrivées. Or, pour répartir les frais fixes et garantir des revenus satisfaisants, la limitation excessive n'est pas la panacée. Quant à la répartition égale des touristes entre les villages, cela n'a jamais constitué un stimulant des initiatives. Des formules moins radicales devraient permettre de réguler aussi bien. Enfin, l'auteur évoque les pratiques touristiques des autochtones.

10 "Le cadre juridique des activités touristiques et non gouvernementales en Antarctique" permet à Anne CHOQUET de commenter le protocole de Madrid, protocole au traité sur l'Antarctique, signé en 1991. Si l'ensemble du texte nous informe clairement du cadre réglementaire et montre que le tourisme ne constitue pas un objet 
spécifique, l'arrière-plan idéologique mérite d'être questionné. Quand un article commence par une affirmation aussi péremptoire que "Si l'Antarctique n'est pas une destination classique, le risque de dérive vers un tourisme de masse est à envisager", on tombe vite dans "le tourisme est une activité légitime"... mais il faut "éviter le tourisme de masse". En quoi le tourisme de masse - il faut entendre ici une fréquentation importante que l'auteur ne définit pas - menace-t-il l'Antarctique ? Entre la méconnaissance profonde du tourisme et la capitulation face aux lieux communs du moment...

11 La dernière contribution du dossier est due à Michael HALL qui, dans un article au titre interminable qui commence par "Changement climatique, authenticité et marketing des régions nordiques..." aborde la question du marketing des destinations. Deux idées principales sont développées. D'une part, le positionnement est fragile car son succès induit sa dilution. En effet, une bonne idée, qui se traduit par un accroissement important des opportunités d'affaires, est aussitôt copiée. L'auteur cite ainsi le succès de la stratégie de Rovaneimi, lieu en Laponie finlandaise qui s'est autoproclamé patrie $\mathrm{du}$ Père Noël. Les innovations matérielles ne sont pas non plus à l'abri, comme l'hôtel de glace de Jukkasjärvi inventé dans les années 1990 et dupliqué depuis. D'autre part, une telle stratégie repose sur sa crédibilité et le réchauffement climatique menace la couverture neigeuse, les élevages de rennes qui ne manqueront pas de migrer plus au nord et la forêt d'épineux, accessoires indispensables à la revendication du "Pèrenoëlnationalisme".

Certes mais, là aussi, s'il n'est pas douteux que toutes les évolutions remettent en cause les positions acquises, l'histoire nous montre que, dans un lieu confronté à un tel défi, les acteurs réagissent, s'engagent dans d'autres aventures que le tourisme ou se saisissent d'autres opportunités à l'intérieur du système touristique. Rappelons que Deauville a surmonté la rupture bain froid-peau blanche/bain chaud-teint hâlé, que Boulogne-sur-Mer est devenue une ville industrielle après avoir été l'une des destinations touristiques les plus mondaines du $\mathrm{XIX}^{\mathrm{e}}$ siècle... Bref le réchauffement climatique mérite des analyses plus fournies que la simple évocation des risques. 